VOLUME 28 (2020) 220-228

DOI: $10.24330 /$ ieja. 768269

\title{
NILPOTENT AND LINEAR COMBINATION OF IDEMPOTENT MATRICES
}

\author{
Marjan Sheibani Abdolyousefi \\ Received: 9 December 2019; Accepted: 18 May 2020 \\ Communicated by Huanyin Chen
}

\begin{abstract}
A ring $R$ is Zhou nil-clean if every element in $R$ is the sum of a nilpotent and two tripotents. Let $R$ be a Zhou nil-clean ring. If $R$ is of bounded index or 2-primal, we prove that every square matrix over $R$ is the sum of a nilpotent and a linear combination of two idempotents. This provides a large class of rings over which every square matrix has such decompositions by nilpotent and linear combination of idempotent matrices.
\end{abstract}

Mathematics Subject Classification (2020): 16U40, 47B33

Keywords: Idempotent matrix, nilpotent matrix, linear combination, Zhou nil-clean ring

\section{Introduction}

Throughout, all rings are associative with an identity. Very recently, Zhou investigated a class of rings in which elements are the sum of a nilpotent and two tripotents that commute (see [7]). We call such ring a Zhou nil-clean ring. Many elementary properties of such rings are investigated in [4].

Decomposition of a matrix into the sum of simple matrices is of interest. In this paper, we consider a linear combination of the form

$$
P=N+c_{1} P_{1}+c_{2} P_{2},
$$

where $N$ is a nilpotent matrix and $P_{1}, P_{2}$ are idempotent matrices and $c_{1}$ and $c_{2}$ are scalars. Such decomposition of matrices over Zhou nil-clean rings is thereby determined in this way. A ring $R$ is of bounded index if there exists $m \in \mathbb{N}$ such that $x^{m}=0$ for all nilpotent $x \in R$. A ring $R$ is 2-primal if its primal radical coincides with the set of nilpotents in $R$ [3]. For instance, every commutative (reduced) ring is 2-primal. Let $R$ be Zhou nil-clean. If $R$ is of bounded index or 2 -primal, we prove that every square matrix over $R$ is the sum of a nilpotent and linear combination of two idempotent matrices. This provides a large class of rings over which every square matrix has such decompositions by nilpotent and linear combination of idempotent matrices. 
We use $N(R)$ to denote the set of all nilpotent elements in $R$. N stands for the set of all natural numbers.

\section{Zhou nil-clean rings}

Definition 2.1. A ring $R$ is a Zhou ring if every element in $R$ is the sum of two tripotents that commute.

The structure of Zhou rings was studied in [6]. We now investigate matrices over Zhou rings. We begin with

Lemma 2.2. Every square matrix over $\mathbb{Z}_{3}$ is the sum of two idempotents and a nilpotent.

Proof. See [5, Lemma 2.1].

Lemma 2.3. Every square matrix over $\mathbb{Z}_{5}$ is the sum of a nilpotent and a linear combination of two idempotent matrices.

Proof. As every matrix over $\mathbb{Z}_{5}$ is similar to a companion matrix, we may assume

$$
A=\left(\begin{array}{cccccc}
0 & & & & & c_{0} \\
1 & 0 & & & & c_{1} \\
& 1 & 0 & & & c_{2} \\
& & & \ddots & & \vdots \\
& & & \ddots & 0 & c_{n-2} \\
& & & & 1 & c_{n-1}
\end{array}\right) .
$$

Case I. $c_{n-1}=0$. Choose

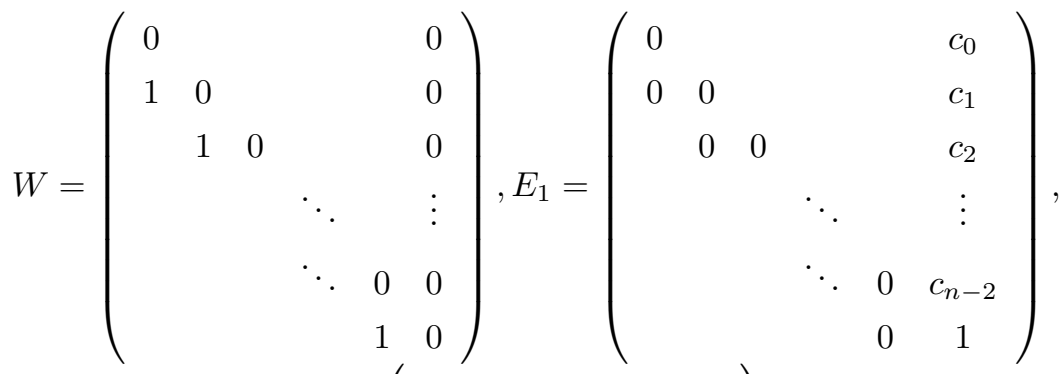

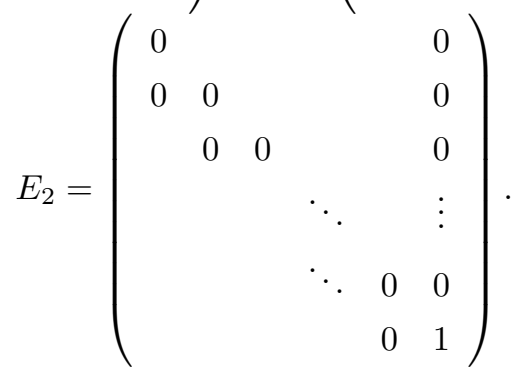


Then $E_{1}^{2}=E_{1}$ and $E_{2}^{2}=E_{2}$, and so $A=E_{1}+(-1) E_{2}+W$.

Case II. $c_{n-1}=1$. Choose

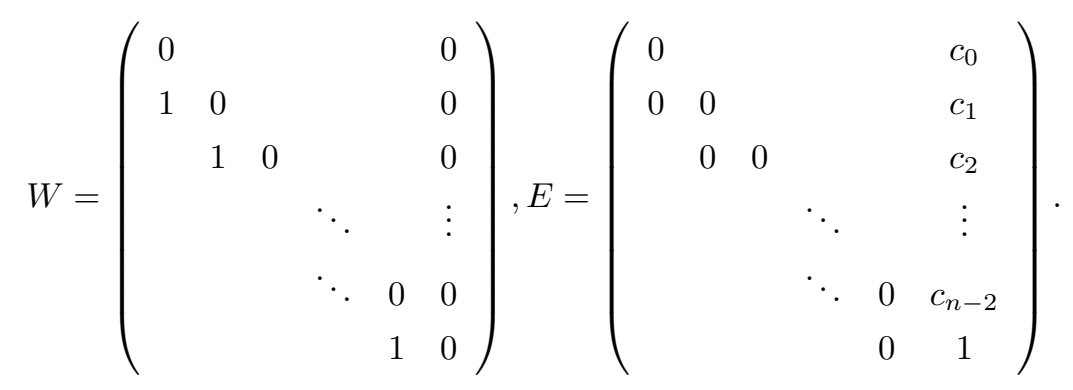

Then $E^{2}=E$, and so $A=E+0+W$.

Case III. $c_{n-1}=-1$. Choose

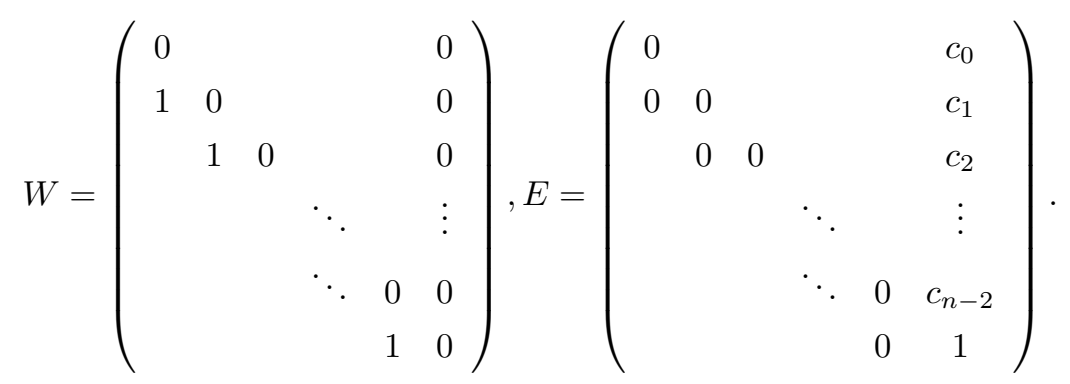

Then $E^{2}=E$, and so $A=(-1) E+0+W$.

Case IV. $c_{n-1}=2$. Choose

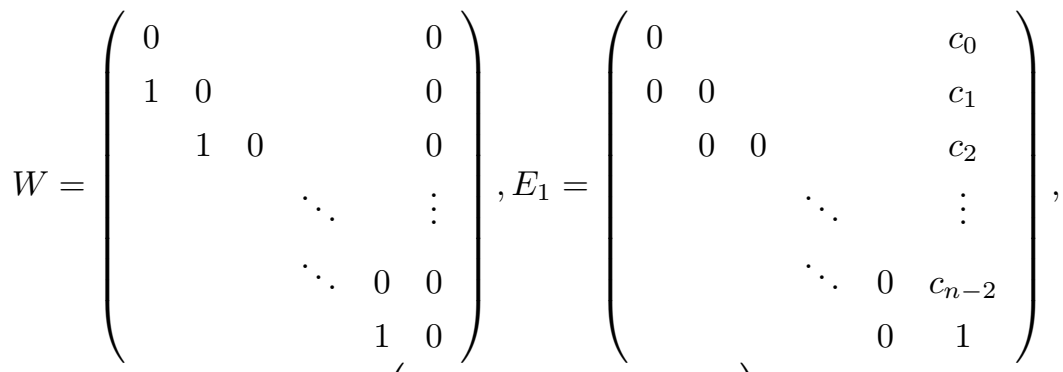

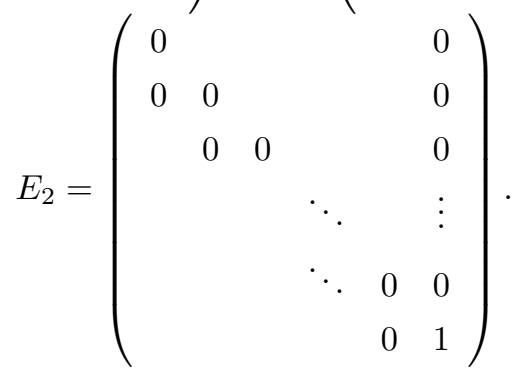

Then $E_{1}^{2}=E_{1}$ and $E_{2}^{2}=E_{2}$, and so $A=E_{1}+E_{2}+W$. 
Case IV. $c_{n-1}=-2$. Choose

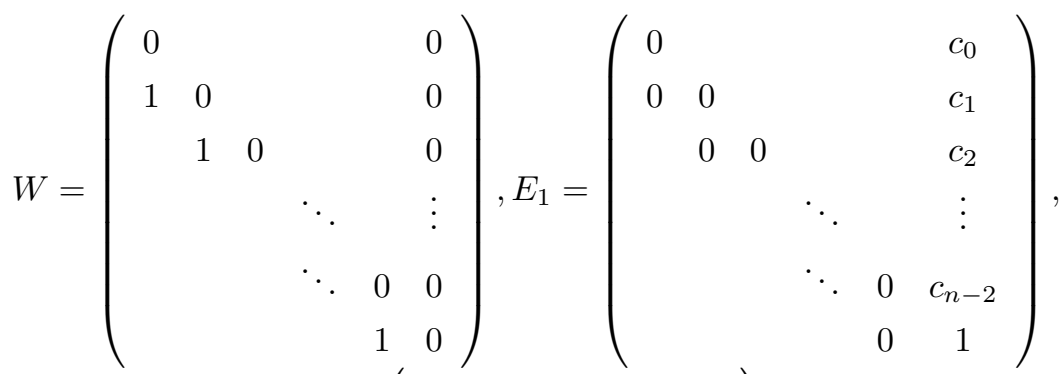

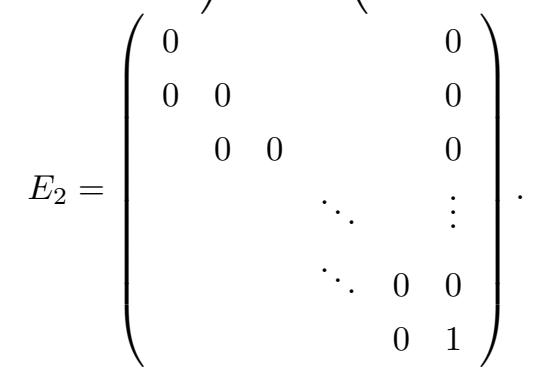

Then $E_{1}^{2}=E_{1}$ and $E_{2}^{2}=E_{2}$, and so $A=(-1) E_{1}+(-1) E_{2}+W$.

Therefore we complete the proof.

Recall that a ring $R$ is a Yaqub ring if it is the subdirect product of $\mathbb{Z}_{3}$ 's. A ring $R$ is a Bell ring if it is the subdirect product of $\mathbb{Z}_{5}$ 's. We have

Lemma 2.4. Every Zhou ring is isomorphic to a strongly nil-clean ring of bounded index, a Yaqub ring, a Bell ring or products of such rings.

Proof. See [5, Lemma 2.3].

Lemma 2.5. (See [2, Lemma 6.6]) Let $R$ be of bounded index. If $J(R)$ is nil, then $J\left(M_{n}(R)\right)$ is nil for all $n \in \mathbb{N}$.

We are ready to prove the following.

Theorem 2.6. Let $R$ be a Zhou nil-clean ring of bounded index. Then every square matrix over $R$ is the sum of a nilpotent and linear combination of two idempotent matrices.

Proof. In view of Lemma 2.3, $R$ is isomorphic to $R_{1}, R_{2}, R_{3}$ or the products of these rings, where $R_{1}$ is a strongly nil-clean ring of bounded index, $R_{2}$ is a Yaqub ring and $R_{3}$ is a Bell ring.

Step 1. Let $A \in M_{n}\left(R_{1}\right)$. In view of [2, Corollary 6.8], there exist an idempotent $E \in M_{n}\left(R_{1}\right)$ and $W \in N\left(M_{n}\left(R_{1}\right)\right)$ such that $A=E+W$. 
Step 2. Let $A \in M_{n}\left(R_{2}\right)$, and let $S$ be the subring of $R_{2}$ generated by the entries of $A$. That is, $S$ is formed by finite sums of monomials of the form: $a_{1} a_{2} \cdots a_{m}$, where $a_{1}, \ldots, a_{m}$ are entries of $A$. Since $R_{2}$ is a commutative ring in which $3=0$, $S$ is a finite ring in which $x=x^{3}$ for all $x \in S$. Thus, $S$ is isomorphic to finite direct product of $\mathbb{Z}_{3}$. As $A \in M_{n}(S)$, it follows by Lemma 2.1 that $A$ is the sum of two idempotents and a nilpotent matrix over $S$.

Step 3. Let $A \in M_{n}\left(R_{3}\right)$, and let $S$ be the subring of $R_{3}$ generated by the entries of $A$. Analogously, $S$ is isomorphic to finite direct product of $\mathbb{Z}_{5}$. As $A \in M_{n}(S)$, it follows by Lemma 2.2 that $A$ is the sum of a linear combination of two idempotents and a nilpotent matrix over $S$.

Let $A \in M_{n}(R)$. We may write $A=\left(A_{1}, A_{2}, A_{3}\right)$ in $M_{n}\left(R_{1}\right) \times M_{n}\left(R_{2}\right) \times M_{n}\left(R_{3}\right)$, where $A_{1} \in M_{n}\left(R_{1}\right), A_{2} \in M_{n}\left(R_{2}\right), A_{3} \in M_{n}\left(R_{3}\right)$. According to the preceding discussion, we obtain the result.

Example 2.7. Let $n \geq 2$ be an integer, if $n=2^{k} 3^{l} 5^{m}$, then every square matrix over $R=\mathbb{Z}_{n}$ is a linear combination of two idempotents and a nilpotent.

Proof. It is obvious by [5, Example 3.5] that $R$ is a Zhou nil-clean ring, also it is clear that $R$ is of bounded index. Then the result follows from Theorem 2.5.

\section{2-Primal rings}

An element $w$ in a ring $R$ is called strongly nilpotent if any chain $x_{1}=x, x_{2}, x_{3}, \ldots$ with $x_{n+1} \in x_{n} R x_{n}$ forces $x_{m}=0$ for some $m \in \mathbb{N}$. Let $P(R)$ be the primal radical of $R$, i.e., the intersection of all prime ideals of $R$. Then $P(R)$ is exactly the set of all strongly nilpotents in $R[1$, Remark 2.8$]$. We derive

Theorem 3.1. Let $R$ be a ring. Then the following are equivalent:

(1) $R$ is 2-primal and Zhou nil-clean.

(2) $a-a^{5} \in R$ is strongly nilpotent for all $a \in R$.

(3) $R / P(R)$ has the identity $x=x^{5}$.

(4) Every element in $R$ is the sum of two tripotents and a strongly nilpotent that commute.

Proof. $(1) \Rightarrow(2)$ This is obvious, as every nilpotent in $R$ is strongly nilpotent.

$(2) \Rightarrow(3)$ Since every strongly nilpotent in $R$ is contained in $P(R)$, we are through.

$(3) \Rightarrow(4)$ Let $a \in R$. Then $\bar{a}=\bar{a}^{5}$; hence, $a-a^{5} \in P(R)$ is nilpotent. Thus, $R$ is Zhou nil-clean. In view of [7, Theorem 2.11], every element in $R$ is the sum of 
two tripotents $u, v$ and a nilpotent $w$ that commute. Write $w^{n}=0(n \in \mathbb{N})$. Then $\bar{w}=\bar{w}^{5 n} \in R / P(R)$. Hence, $w \in P(R)$, i.e., $w$ is strongly nilpotent, as desired.

$(4) \Rightarrow(1)$ As every strongly nilpotent in $R$ is nilpotent, $R$ is Zhou nil-clean, by [7, Theorem 2.11]. In view of [7, Theorem 2.11], $2 \times 3 \times 5 \in N(R)$. Write $2^{n} \times 3^{n} \times 5^{n}=0(n \in \mathbb{N})$. Since $(2,3,5)=1$, by the Chinese Remainder Theorem, $R \cong R_{1} \times R_{2} \times R_{3}$, where $R_{1}=R / 2^{n} R, R_{2}=R / 3^{n} R$ and $R_{3}=R / 5^{n} R$. Step 1. Let $a \in N\left(R_{1}\right)$. Then $a=e+w$ with $e^{3}=e, w \in P(R)$ and $a e=e a$. As $2 \in N\left(R_{1}\right)$, we see that $2 \in P\left(R_{1}\right)$, as it is central. Hence, $a^{2}-a^{4} \in P(R)$, and so $a\left(a-a^{3}\right) \in P(R)$. As $P(R)$ is an ideal, we see that $\left(a-a^{3}\right)^{2} \in P(R)$. Hence, $\left(a^{3}-a^{5}\right)^{2} \in P(R)$. It follows that $\left(a-a^{5}\right)^{2} \in P(R)$. This implies that $a^{2} \in P(R)$. This implies that $e^{2} \in P(R)$, and so $e=e^{3} \in P(R)$. Therefore $a \in P(R)$. Thus, $N(R) \subseteq P(R)$; hence, $R_{1}$ is 2-primal.

Step 2. Let $a \in N\left(R_{2}\right)$. Then $a=e+w$ with $e^{3}=e, w \in P(R)$ and $a e=e a$. As $3 \in N\left(R_{1}\right)$, we see that $3 \in P\left(R_{1}\right)$, as it is central. Hence, $a-a^{3} \in P(R)$. Hence, $a^{3}-a^{5}=a^{2}\left(a-a^{3}\right) \in P(R)$. It follows that $a-a^{5}=\left(a-a^{3}\right)+\left(a^{3}-a^{5}\right) \in P(R)$. This implies that $a \in P(R)$, and so $N(R) \subseteq P(R)$; hence, $R_{2}$ is 2-primal.

Step 3. Let $a \in N\left(R_{3}\right)$. Then there exist two tripotent $e, f \in R$ and a strongly nilpotent $w \in R$ that commute such that $a=e+f+w$. As $5 \in N\left(R_{3}\right)$, we easily see that $5 \in P\left(R_{3}\right)$, as it is central. Hence, $a^{5} \equiv e^{5}+f^{5}(\bmod P(R))$. Hence, $a^{5} \equiv e+f=a$, and so $a \in P(R)$. This shows that $R_{3}$ is 2-primal.

Therefore $R$ is 2-primal, as asserted.

Corollary 3.2. Let $R$ be a ring. Then the following are equivalent:

(1) $R$ is 2-primal and Zhou nil-clean.

(2) Every element in $R$ is the sum of four idempotents and a strongly nilpotent that commute.

Proof. (1) $\Rightarrow(2)$ This is obvious, by [4, Theorem 2.5]. (2) $\Rightarrow(1)$ Let $a \in R$. Then there exist idempotents $e, f, g, h \in R$ and a strongly nilpotent $w \in R$ that commute such that $2-a=e+f+g+h+w$. Hence, $a=(1-e)-f+(1-g)-h-w$. Obviously, $(1-e)-f,(1-g)-h \in R$ are both tripotents. Therefore $a$ is the sum of two tripotents and a strongly nilpotent that commute. According to Theorem 3.1, $R$ is 2-primal and Zhou nil-clean.

Theorem 3.3. Every subring of 2-primal Zhou nil-clean rings is 2-primal Zhou nil-clean.

Proof. Let $S$ be a subring of a 2-primal Zhou nil-clean $R$. For any $a \in S$, we have $a \in R$. By virtue of Theorem 3.1, $a-a^{5} \in P(R)$. 
Given any chain $x_{1}=a-a^{5}, x_{2}, x_{3}, \ldots$ in $S$ with $x_{n+1} \in x_{n} S x_{n}$, we see that this chain is a chain in $R$ with $x_{n+1} \in x_{n} R x_{n}$. Thus, we can find some $m \in \mathbb{N}$ such that $x_{m}=0$. This implies that $a-a^{5} \in S$ is strongly nilpotent. Hence, $a-a^{5} \in P(S)$. By using Theorem 3.1 again, $S$ is a 2-primal Zhou nil-clean ring.

Consequently the center of a 2-primal Zhou nil-clean ring is 2-primal Zhou nilclean. Every corner of 2-primal Zhou nil-clean rings is 2-primal Zhou nil-clean.

Corollary 3.4. Every finite subdirect product of 2-primal Zhou nil-clean rings is 2-primal Zhou nil-clean ring.

Proof. Let $R$ be the subdirect product of 2-primal Zhou nil-clean rings $R_{1}, \ldots, R_{n}$. Then $R$ is isomorphic to the subring of $R_{1} \times \cdots \times R_{n}$. In view of Theorem 5.3, $R$ is a 2-primal Zhou nil-clean ring.

Example 3.5. Let $R$ be a ring. Set $S=\{(x, y) \in R \times R \mid x-y \in J(R)\}$, which is a subring of $R \times R$. Then $R$ is 2-primal Zhou nil-clean if and only if $S$ is 2-primal Zhou nil-clean.

Proof. $\Rightarrow$ Clearly, $S$ is a subring of $R \times R$. Thus, $S$ is 2-primal Zhou nil-clean.

$\Leftarrow$ Since $R$ is a homomorphic image of $S$, we easily obtain the result.

Example 3.6. Let $V$ be a countably-infinite-dimensional vector space over $\mathbb{Z}_{5}$, with $\left\{v_{1}, v_{2}, \ldots\right\}$ a basis, let

$$
A=\left\{f \in \operatorname{End}(V) \mid \operatorname{rank}(f)<\infty, f\left(v_{i}\right) \in \sum_{k=1}^{i} v_{k} \mathbb{Z}_{5} \text { for all } i \in \mathbb{N}\right\} ;
$$

and let $R$ be the $\mathbb{Z}_{5}$-algebra of $\operatorname{End}(V)$ generated by $A$ and the identity endomorphism. Then $R$ is 2-primal Zhou nil-clean.

Proof. In view of [3, Example 4.2.20],

$$
P(R)=\left\{f \in A \mid f\left(v_{i}\right) \in \sum_{k=1}^{i-1} v_{k} \mathbb{Z}_{5} \text { for all } i \in \mathbb{N}\right\},
$$

and then $R / P(R)$ is isomorphic to the ring of all eventually-constant sequences in the direct product of $\mathbb{Z}_{5}^{\prime} s$; hence, $R / P(R)$ has the identity $x=x^{5}$. Therefore $a-a^{5} \in P(R)$ for all $a \in R$. By using Theorem 3.1, $R$ is a 2-primal Zhou nil-clean ring, as asserted.

Proposition 3.7. Let $R$ be a ring. Then the following are equivalent:

(1) $R$ is 2-primal Zhou nil-clean. 
(2) $T_{n}(R)$ is 2-primal Zhou nil-clean for some $n \in \mathbb{N}$.

(3) $T_{n}(R)$ is 2-primal Zhou nil-clean for all $n \in \mathbb{N}$.

Proof. $(1) \Rightarrow(3)$ Let $I=\left\{\left(\begin{array}{cccc}0 & a_{12} & \cdots & a_{1 n} \\ 0 & 0 & \cdots & a_{2 n} \\ \vdots & \vdots & \ddots & \vdots \\ 0 & 0 & \cdots & 0\end{array}\right) \in T_{n}(R) \mid\right.$ each $\left.a_{i i}=0\right\}$. Then $I$ is a nilpotent ideal of $T_{n}(R)$. Since $T_{n}(R) / I \cong \bigoplus_{i=1}^{n} R_{i}$ with each $R_{i}=R$, the finite direct product $\bigoplus_{i=1}^{n} R_{i}$ is Zhou nil-clean. It is obvious that $T_{n}(R)$ is Zhou nil-clean. Let $x \in N\left(T_{n}(R)\right)$. Then $\bar{x} \in N\left(T_{n}(R) / I\right)$. Given any chain $x_{1}=x, x_{2}, x_{3}, \ldots$ in $T_{n}(R)$ with $x_{m+1} \in x_{m} T_{m}(R) x_{m}$, we get a chain $\overline{x_{1}}=\bar{x}, \overline{x_{2}}, \overline{x_{3}}, \ldots$ in $T_{m}(R) / I$ with $\overline{x_{m+1}} \in \overline{x_{m}}\left(T_{m}(R) / I\right) \overline{x_{m}}$. As $\bar{x} \in T_{n}(R) / I$ is strongly nilpotent, we see that $\overline{x_{k}}=\overline{0}$ for some $k \in \mathbb{N}$, i.e., $x_{k} \in I$. Since $I^{n}=0$, we see that $x_{k+n} \in I^{n}=0$, and so $x \in T_{n}(R)$ is strongly nilpotent. Hence, $T_{n}(R)$ is 2-primal, as asserted.

$(3) \Rightarrow(2)$ This is obvious.

$(2) \Rightarrow(1)$ Clearly, $R$ is isomorphic to a subring of $T_{n}(R)$, thus we obtain the result by Theorem 3.3 .

Theorem 3.8. Let $R$ be a 2-primal Zhou nil-clean ring. Then every square matrix over $R$ is the sum of a nilpotent and linear combination of two idempotent matrices.

Proof. Since $R$ is a Zhou nil-clean ring, it follows by [7, Theorem 2.11] that $J(R)$ is nil and $R / J(R)$ has the identity $x=x^{5}$. Hence, $R / J(R)$ is Zhou nil-clean of bounded index 5. By virtue of Theorem 2.5, every matrix in $M_{n}(R / J(R))$ is the sum of a nilpotent and linear combination of two idempotent matrices. Clearly, $J(R) \subseteq N(R)=P(R) \subseteq J(R)$, we have $J(R)=P(R)$. Therefore $M_{n}(J(R))=$ $M_{n}(P(R))=P\left(M_{n}(R)\right)$ is nil. It follows from $M_{n}(R / J(R)) \cong M_{n}(R) / M_{n}(J(R))$ that every matrix in $M_{n}(R)$ is the sum of a nilpotent and linear combination of two idempotent matrices.

Corollary 3.9. Let $R$ be a commutative Zhou nil-clean ring. Then every square matrix over $R$ is the sum of a nilpotent and linear combination of two idempotent matrices.

Proof. Since every commutative ring is 2-primal, we obtain the result by Theorem 3.8 .

Acknowledgement. The author would like to thank the referee for the valuable suggestions and comments. 


\section{References}

[1] P. Danchev, Strongly nil-clean corner rings, Bull. Iranian Math. Soc., 43 (2017), 1333-1339.

[2] M. T. Kosan, Z. Wang and Y. Zhou, Nil-clean and strongly nil-clean rings, J. Pure Appl. Algebra, 220 (2016), 633-646.

[3] G. T. Marks, 2-Primal Rings, Ph.D. Thesis, University of California, Berkeley, 2000.

[4] M. Sheibani Abdolyousefi, N. Ashrafi and H. Chen, On Zhou nil-clean rings, arXiv:1705.05094 [math.RA], (2017).

[5] M. Sheibani Abdolyousefi and H. Chen, Matrices over Zhou nil-clean rings, Comm. Algebra, 46 (2018), 1527-1533.

[6] Z. Ying, M. T. Kosan and Y. Zhou, Rings in which every element is a sum of two tripotents, Canad. Math. Bull., 59 (2016), 661-672.

[7] Y. Zhou, Rings in which elements are sums of nilpotents, idempotents and tripotents, J. Algebra Appl., 17 (2018), 1850009 (7 pp).

Marjan Sheibani Abdolyousefi

Women's University of Semnan (Farzanegan)

Semnan, Iran

e-mail: sheibani@fgusem.ac.ir 